\title{
Full QCD light hadron spectrum from the CP-PACS *
}

\section{CP-PACS Collaboration :}

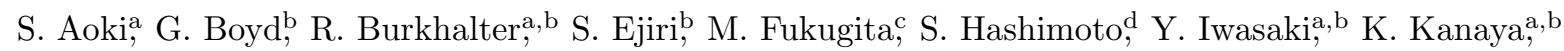
T. Kaneko, Y. Kuramashi, K. Nagai, M. Okawa, H.P. Shanahan, A. Ukawa, ${ }^{\mathrm{a}, \mathrm{b}}$ and T. Yoshiéa,b

${ }^{a}$ Institute of Physics, University of Tsukuba, Tsukuba, Ibaraki 305-8571, Japan

${ }^{\mathrm{b}}$ Center for Computational Physics, University of Tsukuba, Tsukuba, Ibaraki 305-8577, Japan

${ }^{\mathrm{c} I n s t i t u t e}$ for Cosmic Ray Research, University of Tokyo, Tanashi, Tokyo 188-8502, Japan

${ }^{\mathrm{d} H i g h}$ Energy Accelerator Research Organization (KEK), Tsukuba, Ibaraki 305-0801, Japan

We report on an on-going two-flavor full QCD study on CP-PACS using an RG-improved gauge action and a tadpole-improved SW quark action. Runs are made for three lattice spacings $a^{-1} \approx 0.9$, 1.3 , and $2.5 \mathrm{GeV}$ on $12^{3} \times 24,16^{3} \times 32$, and $24^{3} \times 48$ lattices. Four sea quark masses having $m_{\mathrm{PS}} / m_{\mathrm{V}} \approx 0.8-0.6$ are simulated, for each of which hadron masses are evaluated for valence quark masses corresponding to $m_{\mathrm{PS}} / m_{\mathrm{V}} \approx 0.8-0.5$. Results for hadron and light quark masses are presented and compared with those obtained in quenched QCD.

\section{Introduction}

Having observed an unambiguous deviation of the quenched light hadron spectrum from the experiment|1] 3], we have started, as a logical next step, a systematic full QCD simulation. Due to the necessity of working at coarse lattice spacings to cope with an increased demand on computer power, we employ an RG-improved gauge action combined with a meanfield-improved SW quark action. This choice is an outcome of a comparative study of various action combinations carried out prior to the present work 4 . In this report we describe the main points of results obtained so far, referring to Ref. [3] for details.

\section{Parameters of simulation}

We study QCD with two flavors of sea quarks, identified with the degenerate $u$ and $d$ quarks, treating the strange quark in the quenched approximation. Based on test simulations, we choose the run parameters listed in Table 11 corresponding to three lattice spacings in the range $a^{-1} \sim 1-2 \mathrm{GeV}$. We employ $12^{3} \times 24,16^{3} \times 32$, and $24^{3} \times 48$ lattices to keep the spatial size at

\footnotetext{
* presented by K. Kanaya at Lattice98, Boulder, Colorado,
} USA, 13-18 July 1998.
Table 1

Parameters of simulation.

\begin{tabular}{ccccc}
\hline $\begin{array}{c}\text { lattice } \\
\beta / c_{\mathrm{SW}}\end{array}$ & $\begin{array}{c}a^{-1}[\mathrm{GeV}] \\
L a[\mathrm{fm}]\end{array}$ & $K_{\text {sea }}$ & $m_{\mathrm{PS}} / m_{\mathrm{V}}$ & $\begin{array}{c}N_{\text {conf }} \\
\times N_{\text {sepr }}\end{array}$ \\
\hline $12^{3} \times 24$ & $0.917(10)$ & 0.1409 & $0.806(1)$ & $1250 \times 5$ \\
$1.80 / 1.60$ & $2.58(3)$ & 0.1430 & $0.753(1)$ & $1000 \times 5$ \\
& & 0.1445 & $0.696(2)$ & $1400 \times 5$ \\
& & 0.1464 & $0.548(4)$ & $1050 \times 5$ \\
\hline $16^{3} \times 32$ & $1.288(15)$ & 0.1375 & $0.805(1)$ & $1400 \times 5$ \\
$1.95 / 1.53$ & $2.45(3)$ & 0.1390 & $0.751(1)$ & $1400 \times 5$ \\
& & 0.1400 & $0.688(1)$ & $1400 \times 5$ \\
& & 0.1410 & $0.586(3)$ & $1000 \times 5$ \\
\hline $24^{3} \times 48$ & $2.45(9)$ & 0.1351 & $0.800(2)$ & $250 \times 5$ \\
$2.20 / 1.44$ & $1.93(7)$ & 0.1358 & $0.752(3)$ & $270 \times 5$ \\
& & 0.1363 & $0.702(3)$ & $322 \times 5$ \\
& & 0.1368 & $0.637(6)$ & $253 \times 5$ \\
\hline
\end{tabular}

$L \sim 2.4 \mathrm{fm}$. Simulations are made at sea quark masses corresponding to $m_{\mathrm{PS}} / m_{V} \approx 0.8,0.75$, 0.7 and 0.6 .

At each sea quark mass, the light hadron spectrum is computed using valence quark masses corresponding to $m_{\mathrm{PS}} / m_{\mathrm{V}} \approx 0.8,0.75,0.7,0.6$ and 0.5 . We smear the quark source with an exponential smearing function [4. All unequal quark mass combinations allowed for degenerate $u$ and $d$ sea quarks are taken. Hadron masses are ex- 


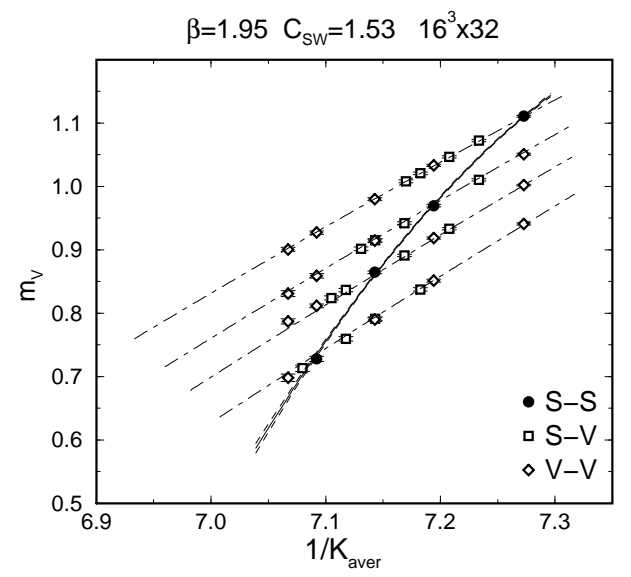

Figure 1. Vector meson mass at $\beta=1.95$. Lines are results of quadratic fit. $\mathrm{S}$ and $\mathrm{V}$ correspond to $K_{\text {val }}=K_{\text {sea }}$ and $\neq K_{\text {sea }}$, respectively.

tracted by uncorrelated fits. Errors are estimated by the jackknife method with a bin size of 10 configurations generated over $50 \mathrm{HMC}$ trajectories.

\section{Hadron spectrum}

In Fig. 1 we plot the vector meson mass at $\beta=1.95$ as a function of the average of $1 / K_{\text {val }}$ of the valence quark pair of the meson. Open points along each dashed lines, which are results of global fits described below, are "partially quenched" results calculated for five values of $K_{\text {val }}$ for a fixed $K_{\text {sea }}$ of sea quark. While each of these mass results are almost linear, their slope show a variation with $K_{\text {sea }}$. Moreover, the full QCD results satisfying $K_{v a l}=K_{\text {sea }}$, which are the four filled points, exhibit a concave curvature. Similar behavior is observed also in other hadrons.

We then make a fit of the mass results with a general quadratic ansatz in $1 / K_{\text {sea }}$ and $1 / K_{\text {val }}$. For pseudoscalar mesons the fit ansatz is

$$
\begin{array}{r}
m_{\mathrm{PS}}^{2}=B_{s} \tilde{m}_{\text {sea }}+B_{v} \bar{m}_{\text {val }}+C_{s} \tilde{m}_{\text {sea }}^{2}+C_{v} \bar{m}_{\text {val }}^{2} \\
+C_{\text {sv }} \tilde{m}_{\text {sea }} \bar{m}_{\text {val }}+C_{12} \tilde{m}_{\text {val }(1)} \tilde{m}_{\text {val }(2)}
\end{array}
$$

where bare quark masses are defined as $\tilde{m}_{\text {sea } / \text { val }(i)}=\left(K_{\text {sea } / \text { val }(i)}^{-1}-K_{c}^{-1}\right) / 2$ with $\bar{m}_{v a l}=$ $\left(\tilde{m}_{v a l(1)}+\tilde{m}_{v a l(2)}\right) / 2$ the average valence quark mass. Similar Ansätze without the $C_{12}$ term are used for vector mesons and decuplet baryons. For

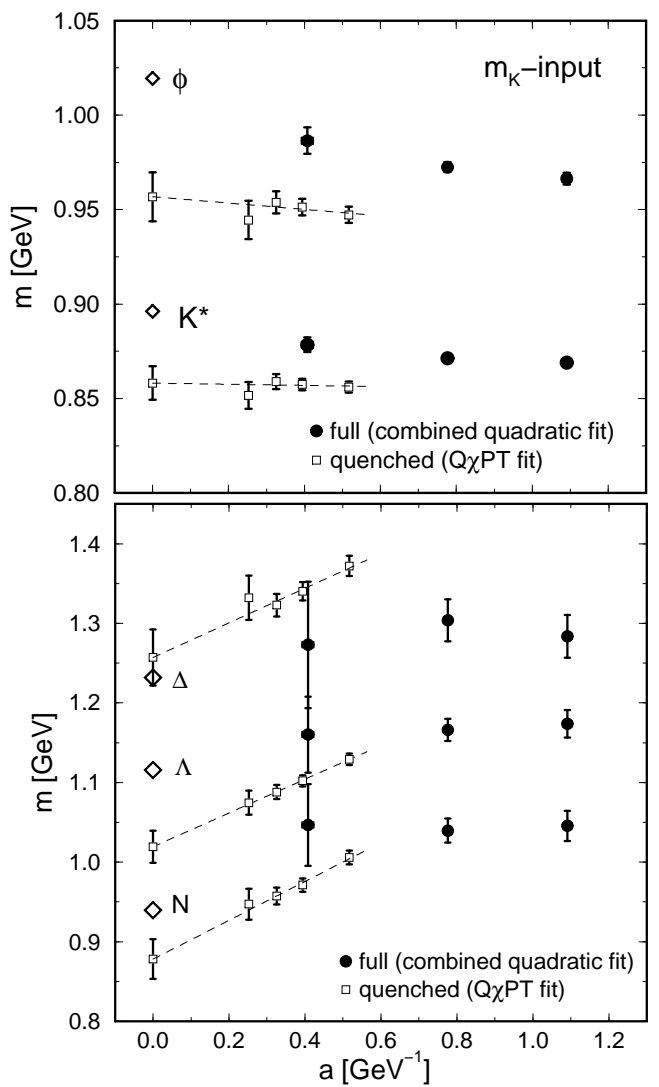

Figure 2. Hadron masses as a function of lattice spacing. Open squares are the results of quenched QCD with Wilson action [2].

octet baryons a form inspired from chiral perturbation theory with general quadratic terms of individual $m_{\text {val }(i)}$ are employed, simultaneously fitting $\Lambda$ and $\Sigma$-like baryons.

From the fit results, we determine the physical light quark point $K_{u d}$ from $m_{\pi} / m_{\rho}$, and the strange quark point $K_{\text {strange }}$ from $m_{K}, m_{K^{*}}$, or $m_{\phi}$. The scale is set by $m_{\rho}$ at $K_{u d}$. Physical hadron masses as a function of the scale are shown in Fig. 2 2 where the results of quenched QCD with the Wilson quark action 2] are also plotted (open symbols).

A very interesting indication in the meson sector is that the present two-flavor full QCD result for the hyperfine splitting extrapolates to a value noticeably closer to experiment than that for quenched QCD in the continuum limit. 


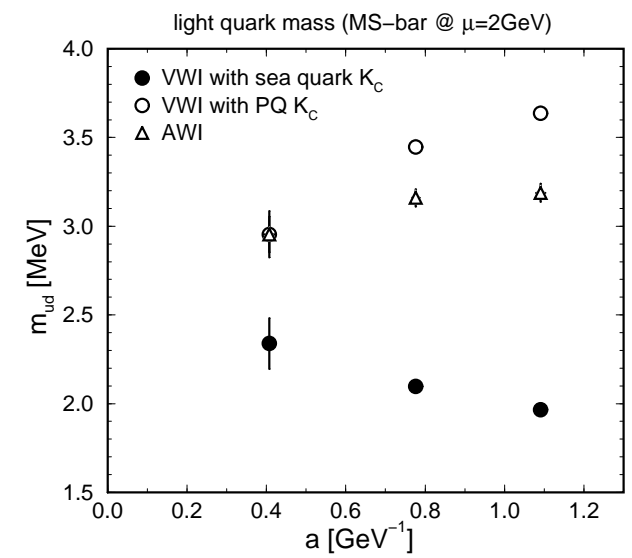

Figure 3. Light quark mass computed with various definitions.

The remaining discrepancy might be due to the quenched treatment of the strange quark itself.

Implications from the baryon results are less clear. While scaling violations are small, the mass results lie $5-10 \%$ high compared to experiment. We note two points in this regard : (i) the lattice size of $2.4 \mathrm{fm}$ (actually turned out to be $1.93 \mathrm{fm}$ at $\beta=2.2$ ) is smaller compared to $3.0 \mathrm{fm}$ for the quenched case, hence finite-size effects can be an issue, and (ii) the smallest quark mass for full QCD corresponds to $m_{P S} / m_{V} \approx 0.5$ rather than $\approx 0.4$ for quenched QCD, possibly leading to an overestimate of baryon masses. Further work matching these points is needed to clarify sea quark effects in the baryon mass spectrum.

\section{Quark masses}

Quark mass can be defined either by vector Ward identity (VWI) $m_{q}^{\mathrm{VWI}}=Z_{m} m_{q}$ with $m_{q}=$ $\left(1 / K_{q}-1 / K_{\text {crit }}\right) / 2$, or by axial Ward identity (AWI) $Z_{A} \nabla_{\mu} A_{\mu}=2 m_{q}^{\mathrm{AWI}} Z_{P} P$. A recent discussion with the VWI definition has been whether to take the critical value $K_{c}^{s e a}$ of sea quark for $K_{\text {crit }}$ or the partially quenched critical value $K_{c}^{\mathrm{PQ}}$ defined by $m_{\mathrm{PS}}\left(K_{\text {val }}\right)=0$ with $K_{\text {sea }}=K_{u d}$ fixed [6, [7. The former is a natural choice for the averaged mass $m_{u d}$ of $u$ and $d$ quarks since sea quarks in two-flavor full QCD are identified with them. The SESAM Collaboration found, however, that this choice leads to a large value of the ratio $m_{s} / m_{u d} \sim 50$ at $a^{-1} \approx 2 \mathrm{GeV}$. The value of $m_{u d}$ itself differs by a factor two depending on the two choices. This effect is negiligibly small for the heavier strange quark.

In Fig. 3 we show our results for $m_{u d}$ at $2 \mathrm{GeV}$ in the MS scheme. Renormalization coefficients are taken from one-loop tadpole-improved perturbation theory [5]. The results for the two choices of $K_{\text {crit }}$ in VWI, while sizably differing at finite lattice spacings, converge to a common value of $2.5-3 \mathrm{MeV}$ in the continuum limit, and so does the AWI quark mass. This value is about $40 \%$ smaller than 4.6(2) $\mathrm{MeV}$ from quenched QCD [2].

The convergence found above also resolves the problem with the ratio $m_{s} / m_{u d}$ for the choice $K_{\text {crit }}=K_{c}^{\text {sea }}$; while we find $m_{s} / m_{u d} \sim 50$ at $a^{-1} \sim 0.8 \mathrm{GeV}$, the ratio decreases with the lattice spacing, converging in the continuum limit towards a value $m_{s} / m_{u d} \approx 25$.

For the strange quark mass itself, the main uncertainty arises from the choice of input to fix $K_{\text {strange. }}$. We find $m_{s} \approx 70$ ( $m_{K}$-input $)-80$ ( $m_{\phi}$-input) $\mathrm{MeV}$ in the continuum limit. Compared to $115(2)\left(m_{K}\right.$-input $)-143(6)\left(m_{\phi}\right.$-input $)$ obtained in quenched QCD [2], the discrepancy, which might be explained by the quenching of strange quark, is smaller, and the values are also smaller.

This work is supported in part by the Grantsin-Aid of Ministry of Education (Nos. 08640404, 09304029, 10640246, 10640248, 10740107). GB, SE, and KN are JSPS Research Fellows. HPS is supported by JSPS Research for Future Program.

\section{REFERENCES}

1. CP-PACS Collab., Nucl. Phys. B (Proc. Suppl.) 60A (1998) 14; ibid. 63 (1998) 161; T. Yoshié, ibid. 63 (1998) 3.

2. CP-PACS Collab., presented by T. Yoshié, these proceedings.

3. R. Burkhalter for CP-PACS Collab., these proceedings.

4. CP-PACS Collab., Nucl. Phys. B (Proc. Suppl.) 60A (1998) 335; ibid. 63 (1998) 221.

5. Y. Taniguchi, these proceedings.

6. SESAM Collab., Phys. Lett. B407 (1997) 290.

7. T. Bhattacharya and R. Gupta, Nucl. Phys. B (Proc. Suppl.) 63 (1998) 95. 